\title{
Dynamics of a parametrically excited simple pendulum
}

Gabriela I. Depetri, Felipe A. C. Pereira, Boris Marin, Murilo S. Baptista, and J. C. Sartorelli

Citation: Chaos 28, 033103 (2018); doi: 10.1063/1.5016819

View online: https://doi.org/10.1063/1.5016819

View Table of Contents: http://aip.scitation.org/toc/cha/28/3

Published by the American Institute of Physics 


\title{
Dynamics of a parametrically excited simple pendulum
}

\author{
Gabriela I. Depetri, ${ }^{1}$ Felipe A. C. Pereira, ${ }^{1}$ Boris Marin, ${ }^{2}$ Murilo S. Baptista, ${ }^{3}$ \\ and J. C. Sartorelli, ${ }^{1, a)}$ \\ ${ }^{1}$ Instituto de Física, Universidade de São Paulo, Caixa Postal 66318, 05315-970 São Paulo, Brazil \\ ${ }^{2}$ Departamento de Física, Faculdade de Filosofia, Ciências e Letras, Universidade de São Paulo, Avenida \\ Bandeirantes, 3900, 14040-901 Ribeirão Preto, Brazil \\ ${ }^{3}$ University of Aberdeen, 05508-900 Aberdeen, United Kingdom
}

(Received 21 November 2017; accepted 15 February 2018; published online 6 March 2018)

\begin{abstract}
The dynamics of a parametric simple pendulum submitted to an arbitrary angle of excitation $\phi$ was investigated experimentally by simulations and analytically. Analytical calculations for the loci of saddle-node bifurcations corresponding to the creation of resonant orbits were performed by applying Melnikov's method. However, this powerful perturbative method cannot be used to predict the existence of odd resonances for a vertical excitation within first order corrections. Yet, we showed that period-3 resonances indeed exist in such a configuration. Two degenerate attractors of different phases, associated with the same loci of saddle-node bifurcations in parameter space, are reported. For tilted excitation, the degeneracy is broken due to an extra torque, which was confirmed by the calculation of two distinct loci of saddle-node bifurcations for each attractor. This behavior persists up to $\phi \approx 7 \pi / 180$, and for inclinations larger than this, only one attractor is observed. Bifurcation diagrams were constructed experimentally for $\phi=\pi / 8$ to demonstrate the existence of self-excited resonances (periods smaller than three) and hidden oscillations (for periods greater than three). Published by AIP Publishing. https://doi.org/10.1063/1.5016819
\end{abstract}

Resonance is the absorption of all the energy delivered by an oscillatory excitation to a dynamical system. There are several examples where resonance plays an important role in our daily lives, such as nuclear magnetic resonance (NMR) in medicine; or for our pleasure in musical instruments producing sounds by resonant tubes as in flutes, or resonant strings as in violins. We study parametric resonance, the phenomenon that describes how resonance occurs when a parameter changes as a response to oscillatory excitation, in a paradigmatic system, the parametrically excited pendulum. We focus our study on the persistence of odd periodic resonances with respect to the parameter range for which they exist. Analysis based on the basin of attraction of the resonant orbits reveals how this persistent behavior depends on the tilt of the pendulum pivot alignment.

\section{INTRODUCTION}

Resonance is the absorption of all the energy delivered by an excitation to an oscillatory system. One example is the absorption of radio frequencies by a quantum system in which there are interactions between a nuclear spin and an external magnetic field, as in Ref. $1, F e_{x} Z n_{1-x} F_{2}$ crystals in Nuclear Magnetic Resonance (NMR), where fluorine nuclei have nuclear spin $I=\frac{1}{2}$. Another example is the absorption of microwaves in Electron Paramagnetic Resonance (EPR) in crystals of nickel hexahydrate salts. In this system, ${ }^{2,3}$ the arrangement of six water molecules defines the crystalline field around the nickel ion, giving rise to different symmetries from cubic to

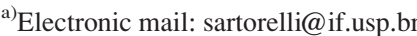

rhombic. In both cases, while the oscillatory excitation frequency $f_{\text {ext }}$ is kept fix, the external magnetic field is varied and resonance occurs when the difference between two energy levels satisfies $\left|E_{j}-E_{k}\right|=\hbar f_{\text {ext }}$. For the first case, NMR, the interactions of the nuclear spin with the neighborhoods do not contribute to the first moment of the resonance curve, while in the second case (EPR), the first moment depends on the crystal field symmetry, which can be lowered, and so, the degeneracy of the levels can be split, and more than one resonance can be observed.

Resonances in plates excited by acoustic waves used as a classical equivalent to quantum chaos, as the Sinai stadium, ${ }^{4}$ for example, are strongly dependent on plate symmetry since the resonance conditions are established by the boundary conditions. Resonant tubes such as woodwind instruments and waves in strings are also examples of resonant systems that depend on the boundary conditions. There are other types of resonances in systems that have fundamental frequencies depending only on physical parameters of the apparatus, such as pendulums and electronic circuits such as RLC, where only one resonance can be observed when the external excitation frequency is equal to the fundamental one.

One paradigmatic system is the parametric simple pendulum. ${ }^{5}$ Parametric resonance occurs when a parameter becomes time dependent as a response to oscillatory excitation. ${ }^{6,7}$ The parametric pendulum presents several resonances with the oscillation frequencies as sub-multiples of the excitation frequency, called subharmonic resonances. Such resonances can be found by determining the occurrence of simple zeros of the Melnikov subharmonic functions to find the loci of saddlenode bifurcations where the subharmonic solutions appear. However, in case the excitation is along the vertical direction, $\phi=0$, odd resonances cannot be determined by the analysis 
of first order corrections of Poincare stroboscopic maps of the system, as showed by Koch and Leven. ${ }^{8}$ This method also cannot determine the occurrence of odd oscillations in other similar symmetric parametric systems, such as a simple pendulum subjected to non-harmonic excitation ${ }^{9}$ and a pendulum with periodically varying lengths. ${ }^{10}$ Using another approach based on Melnikov's method, Kwek and $\mathrm{Li}^{11}$ showed in a very general context that for a nonlinear perturbed system, while even oscillations arise from parametric excitation along the vertical direction, odd oscillations appear as a consequence of external torques. Following this reasoning, one might be lead to conclude that the extra torque that appears when $\phi \neq 0$ is the dynamical mechanism behind the appearance of odd resonances in parametric pendulums. However, Clifford and Bishop, ${ }^{12}$ using symbolic dynamics, reported the occurrence of period-3 oscillations for the vertically excited case, despite being only observable for a small range of control parameter values.

This paper investigates how commonly odd periodic oscillations of symmetric systems should be expected to appear, describing how their behavior changes as we tilt the direction along which the pivot oscillates and what is the dynamical mechanism behind the onset of stable odd resonances. We also present a detailed derivation of the Melnikov subharmonic function for the parametrically excited simple pendulum in the Appendix.

\section{PLANAR SIMPLE PENDULUM REVISITED}

In Fig. 1, a diagram of the experimental apparatus is shown. The harmonic movement of the pendulum pivot in a sliding car is driven by a crank attached to a servo motor. The absolute value of the pendulum angular speed $|\omega|=\left|\frac{d \theta}{d t}\right|$ is obtained with the help of a rotary encoder and the pivot speed $\left|v_{p}\right|=\left|\frac{d s}{d t}\right|$ with a linear encoder. By keeping $A$ constant, the

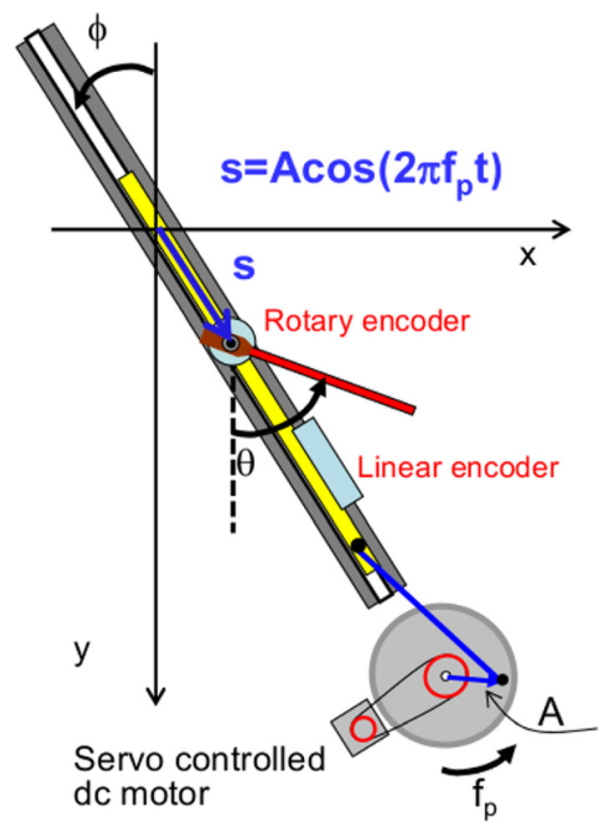

FIG. 1. Diagram representing the experimental apparatus. Fundamental frequency $f_{0}=1.61 \mathrm{~Hz}$, mass $m=37.61 \mathrm{~g}$, center of mass $L_{C M}=5.05 \mathrm{~cm}$ from the pivot position, and dissipation parameter $b=0.0376 \mathrm{~s}^{-1}$. excitation frequency $f_{p}$, in the range $\left[\min \left(f_{p}\right) \max \left(f_{p}\right)\right]$, was spanned first in the forward direction and afterward in the backward direction in steps of $\pm 0.01, \pm 0.05 \mathrm{~Hz}$.

If we write $q=(\theta, \omega)$, then the planar simple pendulum can be described by $\dot{q}=f(q)$, where $f(q)$ is the Hamiltonian vector field

$$
f(\theta, \omega)=\left(\begin{array}{c}
\omega \\
-\sin \theta
\end{array}\right) .
$$

There is a pair of homoclinic orbits $q_{ \pm}^{0}$ that are bi-asymptotic to the saddle points $(\theta, \omega)=( \pm \pi, 0)$. A continuous 1-parameter family of periodic oscillations fills the interior of the homoclinic orbits. This family can be parametrized by a given $\alpha \in(-1,0)$ and can be represented by (Fig. 2)

$$
q^{\alpha}(t)=\left(\theta^{\alpha}(t), \omega^{\alpha}(t)\right),
$$

where $\theta^{\alpha}(t)$ and $\omega^{\alpha}(t)$ are defined implicitly by

$$
\begin{aligned}
\cos \left(\frac{\theta^{\alpha}}{2}\right) & =k \operatorname{sn}(t, k), \quad \sin \left(\frac{\theta^{\alpha}}{2}\right)=\operatorname{dn}(t, k), \\
\omega^{\alpha} & =\dot{\theta}^{\alpha}=2 k \operatorname{cn}(t, k),
\end{aligned}
$$

where $k=\sin \left(\frac{\theta_{\max }^{\alpha}}{2}\right) \in[0,1]$ is the elliptic modulus and $\operatorname{sn}(t, k), \operatorname{cn}(t, k)$, and $\operatorname{dn}(t, k)$ are the Jacobi elliptic functions. ${ }^{13}$ Notice that $T_{\alpha}=4 K(k)$, where $K(k)$ is the complete elliptic integral of first kind, grows monotonically, and tends to infinity as the homoclinic orbit is approached, that is, $T_{\alpha} \rightarrow \infty$ as $\alpha \rightarrow 0$.

\section{PARAMETRIC EXCITATION}

Suppose a pendulum whose pivot oscillates in a tilted direction $\phi$ (for details, see Ref. 7), under linear damping due to viscous dissipation given by $-b \dot{\theta}$. If we define the dimensionless parameters of the system by

$$
\Omega_{p}=\frac{\omega_{p}}{\omega_{0}}, \quad P=\frac{\omega_{p}^{2} A}{g}, \quad \beta=\frac{b}{\omega_{0}},
$$

where $\omega_{0}=2 \pi f_{0}$ is the natural frequency of oscillation of the pendulum and $g$ is the gravitational acceleration, then the equation of motion is given by

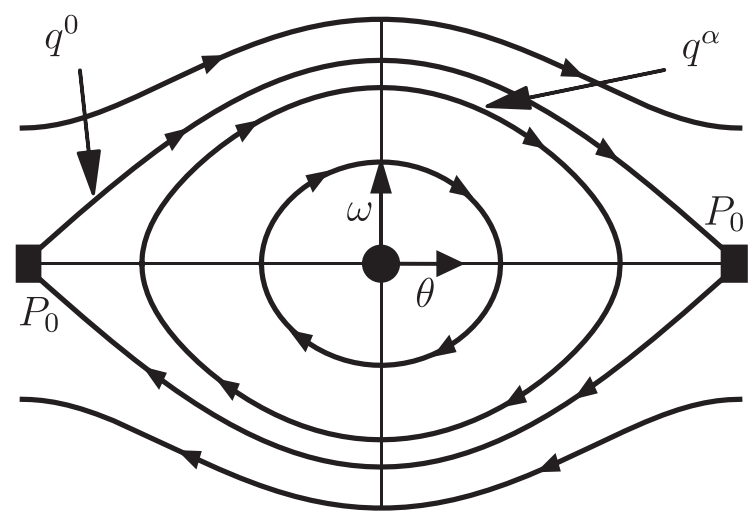

FIG. 2. Phase space of the simple pendulum showing the saddle points $P_{0}$; $q^{\alpha}$ is a family of periodic orbits of period $T_{\alpha}$; and $q^{0}$ is the homoclinic orbit. 


$$
\ddot{\theta}=-\sin \theta-\epsilon\left[P \cos \left(\Omega_{p} t\right) \sin (\theta-\phi)+\beta \omega\right],
$$

where $0<\epsilon \leq 1$. Notice that if $\phi=0$, the pendulum pivot oscillates along the vertical direction and the equations of motion are symmetric with respect to the spatial inversion transformation $\mathrm{R}$

$$
\theta \rightarrow R \theta=-\theta
$$

otherwise, $\phi \neq 0$ adds an extra torque to the movement.

Now, consider the parametrically excited simple pendulum, whose pivot oscillates harmonically along a direction tilted by an angle $\phi$ with the vertical direction, with nondimensionalized amplitude and frequency given by $P$ and $\Omega_{p}$, respectively. If the pendulum is subjected to a viscous dissipation term given by $-\beta \omega$, it can be described by

$$
\dot{q}=f(q)+\epsilon g(q, \dot{q})
$$

and

$$
g\left(\theta, \omega, P, \Omega_{p}, \beta\right)=\left(\begin{array}{c}
0 \\
-P \cos \left(\Omega_{p} t\right) \sin (\theta-\phi)-\beta \omega
\end{array}\right) .
$$

Melnikov's method (see the Appendix for details) states that if the quantity

$$
M^{m}\left(t_{0}\right)=\int_{0}^{m T_{p}} f\left(q^{\alpha}(t), \dot{q}^{\alpha}(t)\right) \times g\left(q^{\alpha}(t), \dot{q}^{\alpha}(t), t\right) \mathrm{d} t
$$

has simple zeros, that is, $M^{m}=0$ but $\frac{\partial M^{m}}{\partial t_{0}} \neq 0$, there will be periodic orbits of period $T_{\alpha}=m T_{p}$, where $m$ is even. Therefore, we obtained the loci of saddle-node bifurcations for even resonances $P_{\mathrm{even}}^{\mathrm{m}}=R_{\mathrm{even}}^{\mathrm{m}}$

$$
R_{\mathrm{even}}^{\mathrm{m}}\left(\Omega_{p}\right)=\frac{4 \beta\left[E(k)-k^{\prime 2} K(k)\right]}{\pi \Omega_{p}^{2} \cos \phi} \sinh \left(\Omega_{p} K^{\prime}(k)\right),
$$

where $k$ is the elliptic modulus, $E(k)$ is the complete elliptic integral of the second kind, $K^{\prime}(k)=K\left(k^{\prime}\right)$, and $k^{\prime 2}=1-k^{2}$ is the complementary elliptic modulus, in accordance with the results of Koch and Leven. ${ }^{8}$ If $m$ is odd, $M^{m}\left(t_{0}\right)$ can only have simple zeros if $\sin \phi \neq 0$, that is, if the additional torque in Eq. (A8) is non-identically null. Then, the loci of saddle-node bifurcations are given by $P_{\mathrm{odd}}^{\mathrm{m}}=R_{\mathrm{odd}}^{\mathrm{m}}$

$$
R_{o d d}^{m}\left(\Omega_{p}\right)=\frac{4 \beta\left[E(k)-k^{\prime 2} K(k)\right]}{\pi \sin \phi\left\{\left[\frac{2 E(k)}{K(k)}-1\right] \operatorname{sech}\left(\Omega_{p} K^{\prime}(k)\right)+\frac{\pi^{2} S}{K^{2}(k)}\right\}},
$$

where $S$ is given by Eq. (A13). Notice that the elliptic modulus $k$ is determined from $\Omega_{p}$ through the resonance condition, and it is worth noticing that for $\Omega_{p}>m$, there is no $k$ such that Eq. (A3) is satisfied. However, oscillations of odd periods are not predictable for vertical excitation due to the fact that odd and even oscillatory solutions exhibit different symmetry properties. While even oscillations are R-invariant [invariant under transformation (5)], odd oscillations are not: they appear in pairs that are R-conjugate. ${ }^{14}$ This difference causes the vanishing of the term that could lead to the occurrence of simple zeros in the Melnikov subharmonic functions for odd oscillations.

\section{RESULTS AND DISCUSSION}

\section{A. Vertical excitation $\phi=0$}

\section{Period-3 oscillations}

When we have a pure vertical parametric excitation, odd periodic resonances cannot be predicted by Melnikov's subharmonic functions due to the symmetry of the equations of motion. Period-3 oscillations were believed to be observable only for a small interval of parameter values, ${ }^{12}$ but we will show that this is not the case. In Fig. 3(a) (parameters $A=2.02 \mathrm{~cm}$ and $f_{p}=5 \mathrm{~Hz}$ ), we show the basins of attraction
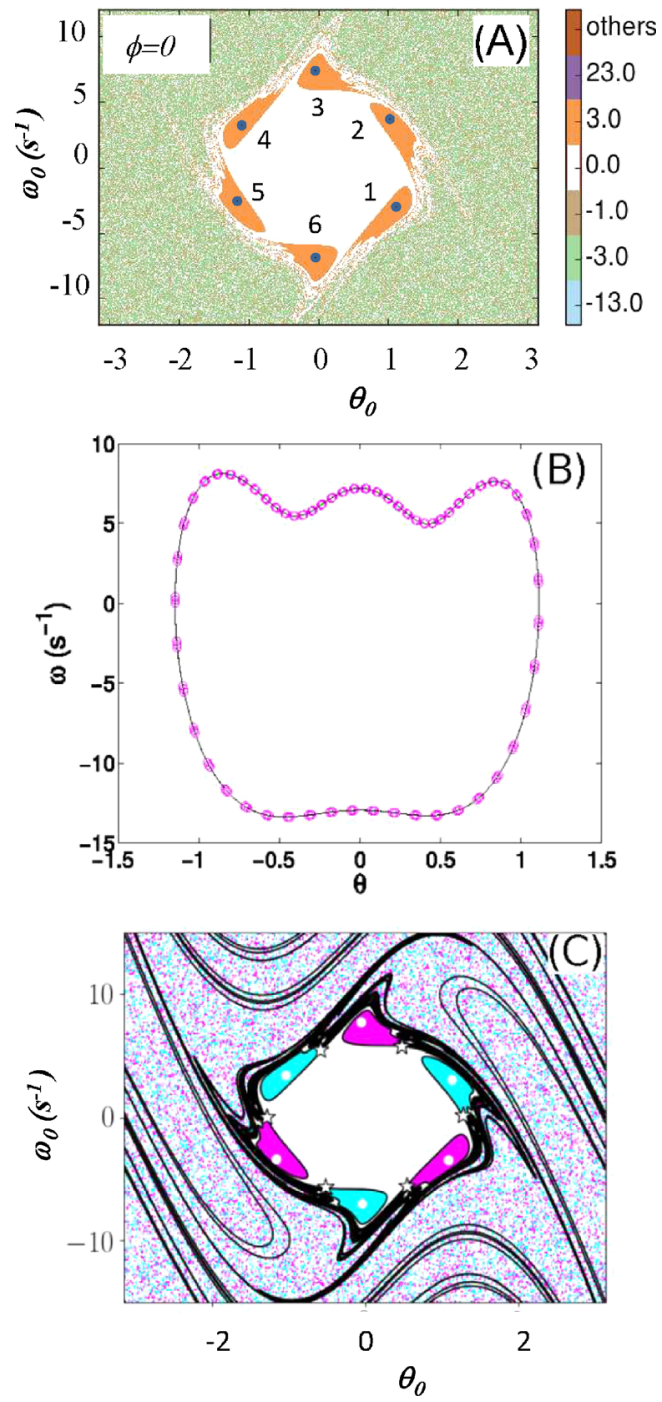

FIG. 3. For $A=2.02 \mathrm{~cm}, f_{p}=5 \mathrm{~Hz}$ and $\phi=0$. (a) The basins of attraction of periodicity as identified by the color bar at right. There are six islands corresponding to period-3 oscillations. Negative numbers indicate periodic rotations. (b) The phase spaces for the six period-3 attractors, where we plotted the odd trajectories $\left(\theta_{\text {odd }}, \omega_{\text {odd }}\right)$ in black and the even ones $\left(-\theta_{\text {even }},-\omega_{\text {even }}\right)$ in purple. In (c), we have the phase basin of attraction for period-3, with unstable fixed points represented by white circles, saddle points by white stars, and in black lines the stable manifolds of the saddle points. 
of a fixed point, period-3, and period-23 oscillations, as well as that of some periodic rotations. In brown, we can see six islands representing the basin of attraction of period- 3 oscillations. An island is a continuous region (open set) in phase space. Integrating the system from the initial conditions labeled with odd numbers in Fig. 3(a) (1, 3, and 5), we obtained the same attractor (henceforth called the odd attractor), while initial conditions 2, 4, and 6 lead to a second attractor (henceforth called the even attractor). In Fig. 3(b), we plotted the phase portraits of the odd attractor $\left(\theta_{\text {odd }}\right.$, $\left.\omega_{\text {odd }}\right)$ as black lines and the even one $\left(-\theta_{\text {even }},-\omega_{\text {even }}\right)$ as circles, showing that they are conjugated by inversion symmetry. For both period-3 attractors, we also constructed basins of attraction by observing the number of different maxima of the $\omega$ time series. If three positive values were found, the point was colored cyan, while for two positive maxima (corresponding to three negative minima), the point was colored magenta, as shown in Fig. 3(c). The black lines show the separatrices between the basins of attraction of
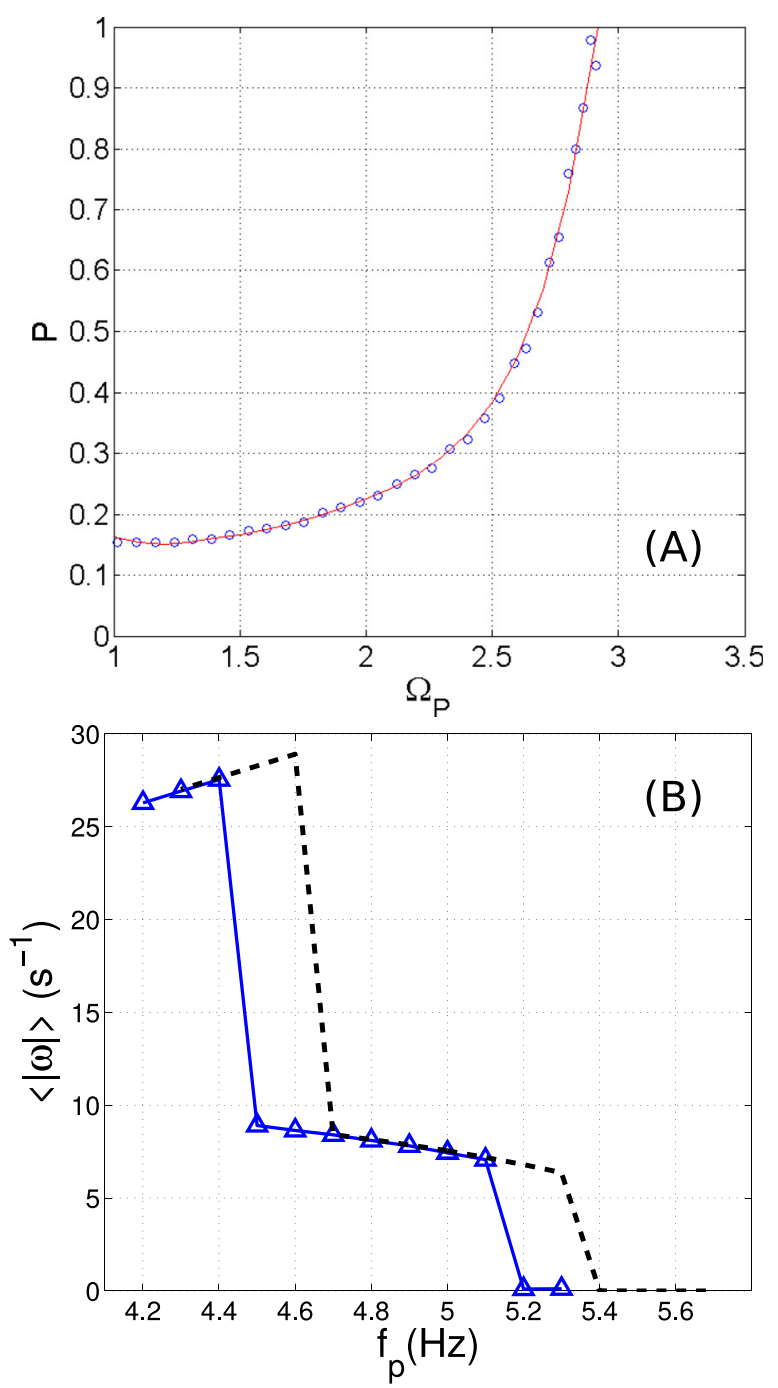

FIG. 4. For $A=2.02 \mathrm{~cm}, f_{p}=5 \mathrm{~Hz}$ and $\phi=0$. In (a), we show that the loci of saddle-node bifurcations are the same for all the six initial conditions, showing that the attractors are indeed degenerate. These lines were obtained by numerical continuation. ${ }^{15}$ (b) Both the experimental (symbols) and the simulated (dashed line) bifurcation diagrams. period-3 oscillations and of equilibrium. They are defined by the stable manifold of the period-3 saddle points that were created together with the period-3 attractors in saddle-node bifurcations.

We call the odd and even attractors shown in Fig. 3(b) degenerate because the loci of saddle-node bifurcations where the attractors are created are the same, as shown in Fig. 4(a). These lines were obtained via numerical continuation. ${ }^{15}$ This degeneracy appears as a result of the spatial inversion symmetry of the system.

Due to the small sizes of the six islands, it is to be expected that finding an initial condition inside the basin of attraction of period-3 experimentally would be very difficult. However, after dozens of tentatives, we managed to find period-3 resonances experimentally for $\phi=0$. We obtained the experimental bifurcation diagram $\langle|\omega|\rangle$ vs. $f_{p}$, where $\langle|\omega|\rangle$ is the mean value of the angular speed, as shown in Fig. 4(b). Experimental data are shown in triangles, while the dashed line was obtained by numerical integration of Eq. (4). It is remarkable not only that the attractors are degenerate, in the sense that they necessarily coexist, but also that they are robust over a small frequency range of $0.7 \mathrm{~Hz}$.

\section{Period-5 oscillations}

In Fig. 5, the basins of attraction for $A=2.02 \mathrm{~cm}$ and $f_{p}=8.55 \mathrm{~Hz}$ are shown. There are ten islands that lead to period-5 oscillations (red), and embedded in each one of these islands, there are other five tiny islands corresponding to the period-25 attractor (green). The large regions in brown are related to the basin of the period-4 attractor, and so, it is very easy to obtain period-4 oscillations experimentally. However, the small size of the islands corresponding to period-5 suggests that such oscillations should be difficult to observe experimentally. In fact, we did not succeed despite having tried dozens of times. Taking into consideration the existence of noise, we conclude that obtaining period-5 oscillations experimentally is practically impossible.

\section{B. Small tilted excitation $\phi=4 \frac{\pi}{180}$}

Breaking the system symmetry by increasing the tilt in the pivot motion to $\phi=4 \frac{\pi}{180}$, we can see that the six islands

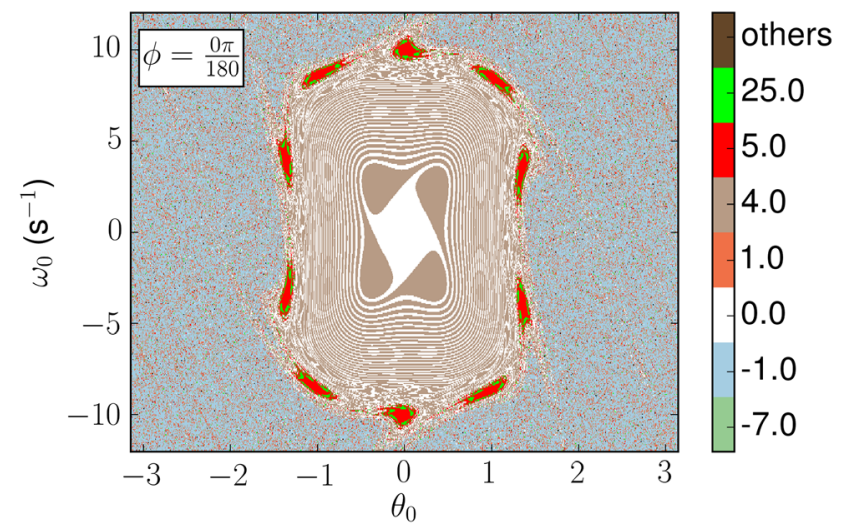

FIG. 5. For $A=2.02 \mathrm{~cm}, f_{p}=8.55 \mathrm{~Hz}$ and $\phi=0$. Basins of attraction of periodicity. The period of each attractor is indicated by colors (color bar on the right). Negative numbers correspond to periodic rotations. 
shown in Fig. 3(a) collapsed into the three islands shown in Fig. 6(a). The large basin of attraction of the fixed point $(0,0)$ was replaced by the basin of period-1 oscillations. As the islands apparently suggest a reflection symmetry $\omega \rightarrow-\omega$, we also chose six initial conditions [numbered from one to six in Fig. 6(a)] to compare with the vertical case. In Fig. 6(b), we show the phase spaces for the six period-3 orbits, where we plotted the three equal odd trajectories in black lines $\left(\omega_{\text {odd }}\right.$ Vs. $\left.\theta_{\text {odd }}\right)$ and in purple the three even trajectories $\left(-\omega_{\text {even }}\right.$ vs. $\left.\theta_{\text {even }}\right)$. Two distinct (non-degenerate) period-3 attractors were obtained. In Fig. 6(c), we can see the basin of attraction of period-3, showing that each island of periodicity is divided into two new regions, now corresponding to different period-3 attractors. These basins are separated by the stable manifold of a period-3 saddle (shown as a white star) that was created together with a period-3 attractor. The period-3 basins suffer an interesting qualitative change, from
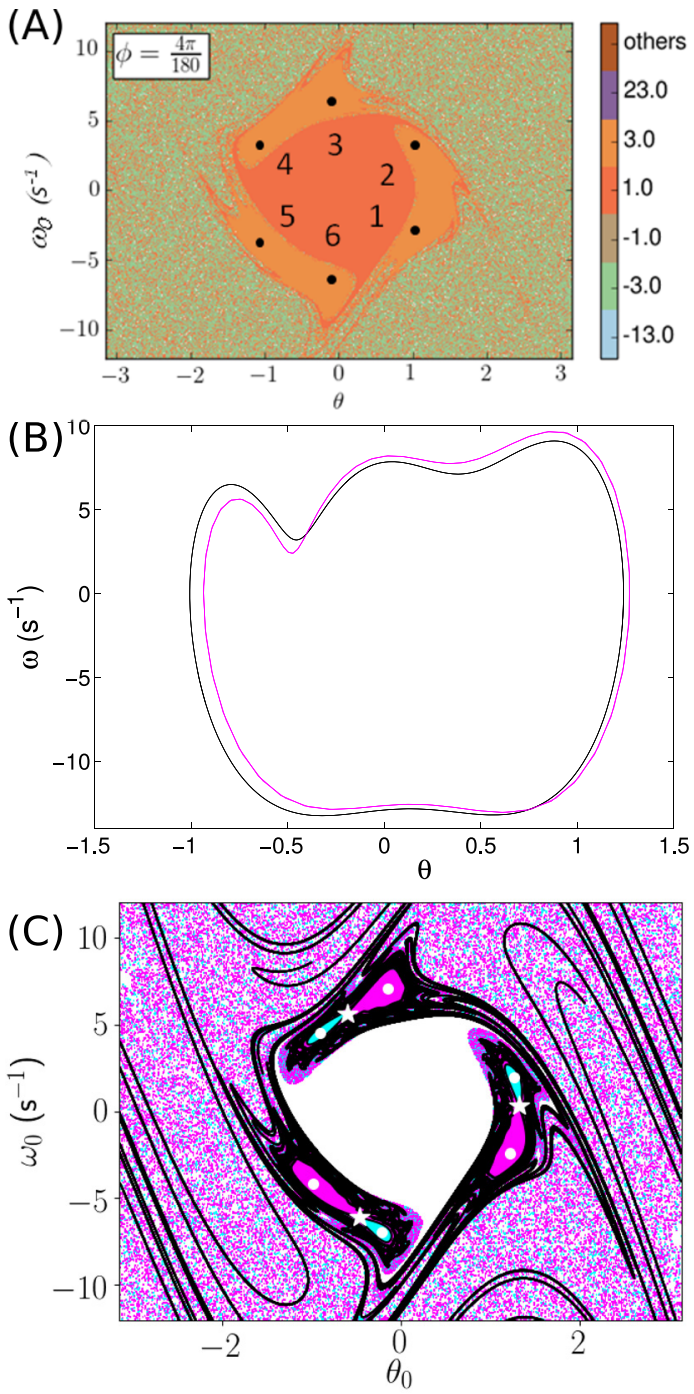

FIG. 6. For $A=2.02 \mathrm{~cm}, f_{p}=5 \mathrm{~Hz}$ and $\phi=4 \frac{\pi}{180}$. In (a), the periodicity basin of attraction identifies three period- 3 islands. (b) The phase portraits for the six initial conditions inside the period-3 basin, where we plotted odd trajectories in black $\left(\omega_{\text {odd }}\right.$ vs. $\left.\theta_{\text {odd }}\right)$ and even ones in magenta $\left(-\omega_{\text {even }}\right.$ vs. $\left.\theta_{\text {even }}\right)$, showing two non-degenerate period-3 attractors. In (c), we have the basin of attraction for period-3. White circles correspond to unstable fixed points, while saddles are indicated by white stars. The stable manifolds of the saddle points are depicted in black. six separated islands to three. The two attractor basins merged in three large period-3 basins due the change in one period-3 saddle position: at $\phi=0$, the stable manifolds of the two period-3 saddles separate the fixed point and one period-3 attractor; at $\phi=4 \frac{\pi}{180}$, one of them separates the period-1 attractor from a period-3 one and the other one separates the two non-degenerate period-3 attractors.

In Fig. 7, we show the loci of saddle-node bifurcations for each of the two (even and odd) period-3 attractors, obtained via numerical continuation. Due to the symmetry break, they cannot be transformed into each other by spatial reversion and the loci of saddle-node bifurcations are not degenerate. Both attractors can still be found up to $\phi \approx 7 \pi / 180$.

Above $\phi \approx 7 \pi / 180$, only a single type of period-3 attractor is observed. Its basin of attraction is composed of three disjoint regions.

\section{Tilted excitation $\phi=\frac{\pi}{8}$}

Experimental bifurcation diagrams were obtained by changing $f_{p}$ at instants $t_{\text {step }}$. This way, the initial conditions after each step are given by $\left(\theta\left(t_{\text {step }}\right), \omega\left(t_{\text {step }}\right)\right)$. After a transient time, the new steady state is recorded.

In the $f_{p}$ range $[0.5,12.1] \mathrm{Hz}$, we measured $\langle|\omega(t)|\rangle$, first in a forward sweep of the excitation frequency, followed by a backward sweep to investigate the self-excited oscillations. By plotting $\langle|\omega|\rangle$ vs. $f_{p}$, we constructed the bifurcation diagrams shown in Fig. 8(a), where circles represent the forward span and a continuous blue line the backward span. By starting at $f_{p}=0.5 \mathrm{~Hz}$, the pendulum oscillates with the same frequency as the excitation as it is increased. As we get closer to $f_{p}=0.8 \mathrm{~Hz}$, the pendulum starts to oscillate with period $T=T_{p} / 2$. Afterward, it resumes oscillating with $T=T_{p}$. Therefore, a bubble of bifurcation $T_{p} \rightarrow T_{p} / 2 \rightarrow T_{p}$ was observed without hysteresis.

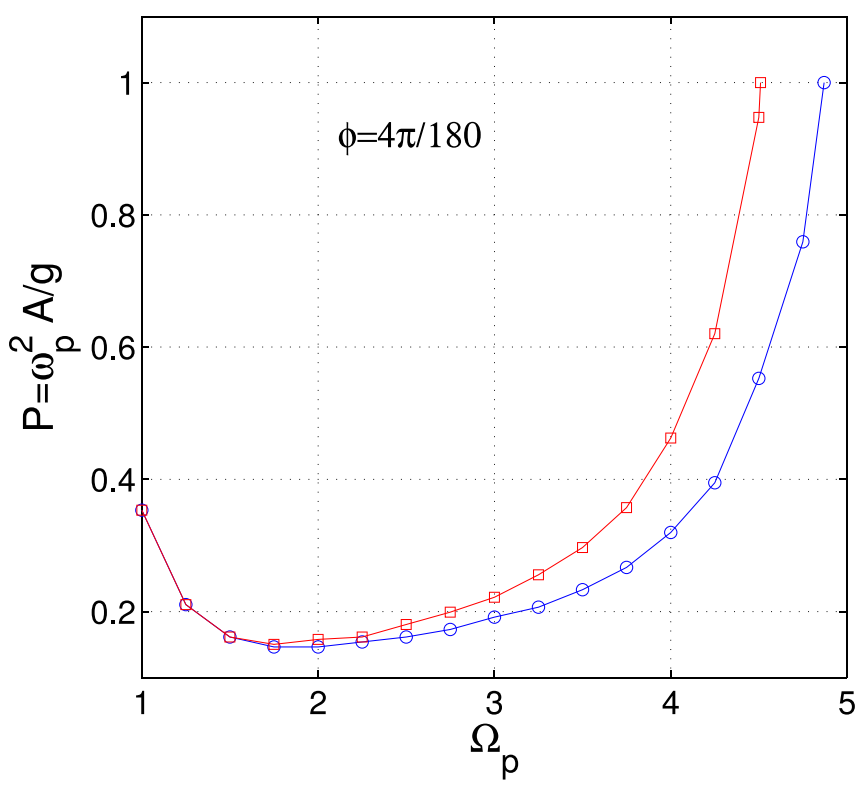

FIG. 7. Loci of saddle-node bifurcations for the two period-3 attractors for $\phi=4 \pi / 180$. After breaking the symmetry, the attractors cease to be degenerate, which is confirmed by the distinct loci of saddle-node bifurcations. 


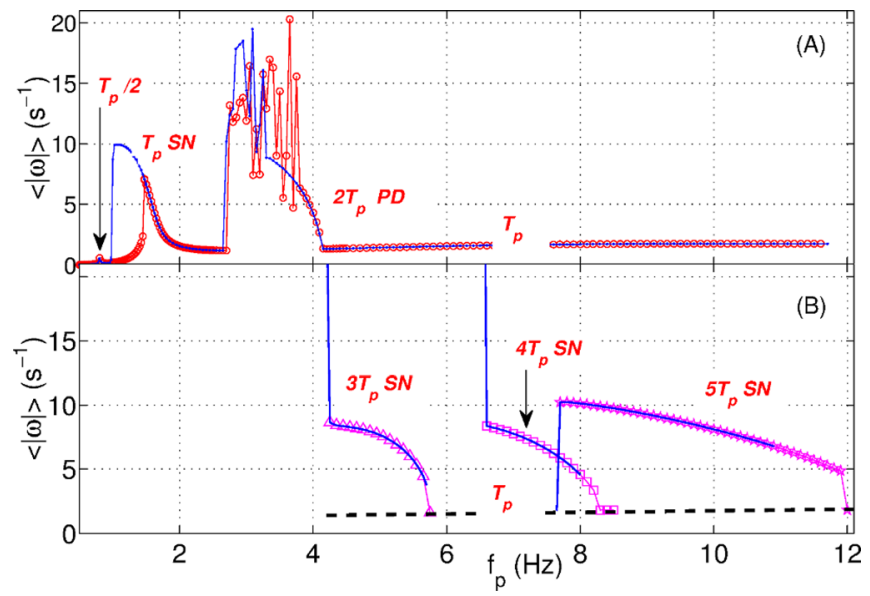

FIG. 8. For $\phi=\frac{\pi}{8}$ and $A=2.02 \mathrm{~cm}$. (a) An experimental bifurcation diagram in the $f_{p}$ interval $[0.5 ; 12.1] \mathrm{Hz}$, showing the self-excited $T=T_{p} / 2, T=T_{p}$, and $T=2 T_{p}$ resonances, related to bifurcation bubble, saddle-node, and period-doubling bifurcations. Above $f_{p} \approx 4.15 \mathrm{~Hz}$, the forced oscillations are due to the additional extra torque given by the tilted excitation. In (b), the experimental bifurcation diagrams for the hidden oscillations $T=3 T_{p}$, $T=4 T_{p}$, and $T=5 T_{p}$ are shown.

We can also observe a $T=T_{p}$ resonance related to a saddle-node bifurcation, clearly presenting hysteresis but preserving the periodicity. After the $f_{p}$ range $[\approx 2.7 ; \approx 3.8]$ $\mathrm{Hz}$ of non-periodic behaviors, we have periodic oscillations related to a period doubling bifurcation, associated with a $T=2 T_{p}$ resonance in the range $[\approx 3.8 \approx 4.15] \mathrm{Hz}$. For $f_{p}$ $>4.15 \mathrm{~Hz}$, the pendulum oscillates with frequency $f=f_{p}$ or $T=T_{p}$, as expected for a forced pendulum with the extra torque due the tilted direction of excitation.

Given that we did not have to search for adequate initial conditions manually, as the experimental apparatus sets them automatically at each $f_{p}$ value, the resonances described in 8(A) are called self-excited. ${ }^{16}$ In Fig. 8(b), the bifurcation diagrams related to saddle-node bifurcations with $T=3 T_{p}, T=4 T_{p}$, and $T=5 T_{p}$ oscillations are shown. Symbols correspond to a forward frequency span, while continuous lines to a backward sweep. For every diagram, we had to look for specific initial conditions $\left(\theta_{0}, \omega_{0}\right)$. The need to specify initial conditions manually is a characteristic of the so-called hidden oscillations.

In Fig. 9, a region of the parameter space $P \times \Omega_{p}$ related to the saddle-node bifurcations shown in Fig. 8(b) is shown. The continuous lines indicated by SN3, SN4, and SN5 are the loci of saddle-node bifurcations and were obtained via numerical continuation. ${ }^{15,17}$ They constitute the borders of primary subharmonic resonance regions $\Omega_{p}=3,4$, and 5, respectively. The full circles, indicated by $R_{\text {odd }}^{3}$ (blue), $R_{\text {even }}^{4}$ (red), and $R_{\text {odd }}^{5}$ (green), are the loci of saddle-node bifurcations predicted by the Melnikov subharmonic functions [See Eqs. (9) and (10)].

The agreement between numerical and analytic predictions for the loci of saddle-node bifurcations (where the resonant periodic attractors of periods-3, 4, and 5 appear for $P<0.5)$ is remarkable.

\section{CONCLUSIONS}

We have studied the parametrically excited pendulum experimentally, numerically, and analytically. For the vertical

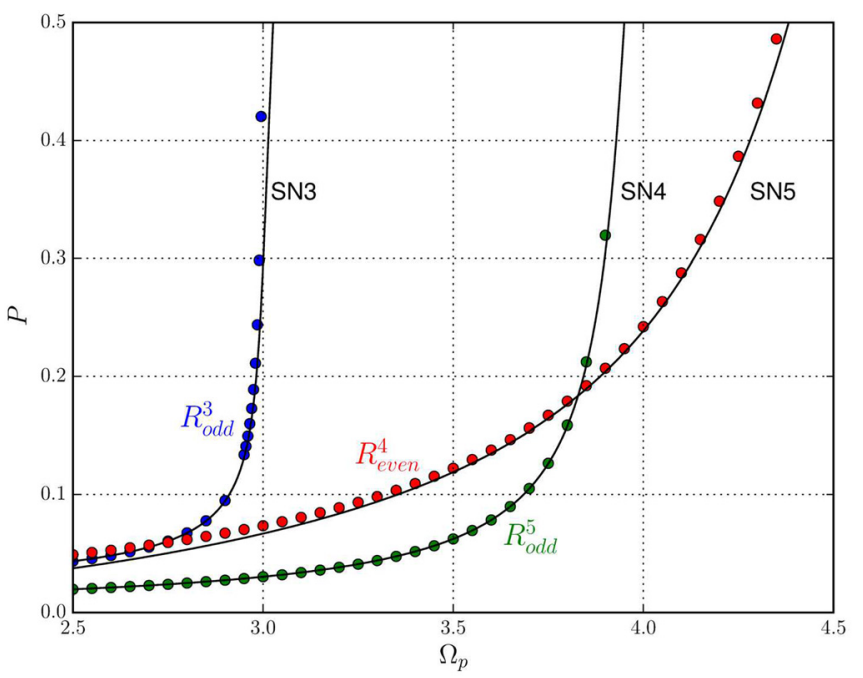

FIG. 9. Parameter space for the tilted parametric pendulum with $\phi=\frac{\pi}{8}$. Continuous lines SN3, SN4, and SN5 are the loci of saddle-node bifurcations obtained by numerical continuation, while full circles indicate $R_{\text {odd }}^{3}$, $R_{\text {even }}^{4}$, and $R_{\text {odd }}^{5}$ obtained by Melnikov's method.

excitation case ( $\phi=0$, a symmetrical system), the existence of odd periodic oscillations is not predictable by Melnikov's method due to the fact that odd and even oscillatory solutions exhibit different symmetry properties. While even oscillations are R-invariant, odd oscillations are not, appearing in Rconjugate pairs. This difference causes the term that could lead to the occurrence of simple zeros in the Melnikov subharmonic functions to vanish for the odd case.

We have characterized two symmetrical period-3 attractors, which were called degenerate as they were created in the same locus of saddle-node bifurcations in parameter space. Despite not being predicted by Melnikov's method (first order), we were able to observe period-3 oscillations experimentally.

We were not able to detect period-5 oscillations experimentally for $\phi=0$ since the corresponding basin of attraction is too small, as demonstrated numerically.

For a small tilted direction $\phi=4 \pi / 180$, the system is not symmetrical anymore, and so, the attractors are not degenerate, which is corroborated by the observation of two distinct loci of saddle-node bifurcations in parameter space. These loci were obtained via both numerical continuation and Melnikov's method, in good agreement. As the basin of attraction of period-3 oscillations composed of large islands, such oscillations were easily observed experimentally.

Taking into account experimentally obtained bifurcation diagrams for $\phi=\pi / 8$, we analyzed self-excited resonances of the period smaller than three, as well as hidden oscillations for periods greater than three, which are related to subharmonic resonances.

\section{ACKNOWLEDGMENTS}

This work was supported by the Brazilian agencies FAPESP (Nos. JCS 2011/19296-1, FACP 2014/07043-0, and BM 2017/04748-0) and CNPq (No. JCS 307947/2014-9). 


\section{APPENDIX: MELNIKOV SUBHARMONIC FUNCTION METHOD}

Consider the parametrically excited simple pendulum, whose pivot oscillates harmonically along a direction tilted by an angle $\phi$ with the vertical direction, with nondimensionalized amplitude and frequency given by $P$ and $\Omega_{p}$, respectively. If the pendulum is subject to a dissipation term given by $-\beta \omega$, it can be described by

$$
\dot{q}=f(q)+\epsilon g(q, \dot{q}),
$$

where $0<\epsilon \leq 1$ and $f(q)$ is the Hamiltonian vector field and

$$
g\left(\theta, \omega, P, \Omega_{p}, \beta\right)=\left(\begin{array}{c}
0 \\
-P \cos \left(\Omega_{p} t\right) \sin (\theta-\phi)-\beta \omega
\end{array}\right) .
$$

\section{Melnikov subharmonic function method}

From the family of periodic oscillations in the interior of the homoclinic orbits, $q^{\alpha}(t)$, consider those satisfying the resonance condition $T_{\alpha}=m T_{p}$, that is,

$$
4 K(k)=m \frac{2 \pi}{\Omega_{p}} .
$$

They are called subharmonic solutions. If $M^{m}\left(t_{0}\right)$, a path integral given by

$$
M^{m}\left(t_{0}\right)=\int_{0}^{m T_{p}} f\left(q^{\alpha}(t), \dot{q}^{\alpha}(t)\right) \times g\left(q^{\alpha}(t), \dot{q}^{\alpha}(t), t\right) \mathrm{d} t,
$$

with $f \times g=f_{1} g_{2}-f_{2} g_{1}$, has simple zeros, then the perturbed system (A1) also has a solution that is a periodic subharmonic oscillation of period $m T_{p}$, arising from a saddle-node bifurcation. ${ }^{18,19}$

The idea is that the quantity $M^{m}\left(t_{0}\right)$ in Eq. (A4) is related to the first order correction of the $m$-th Poincare stroboscopic map of the system. If the unperturbed system has a periodic solution of period $m$, that is, the $m$-th stroboscopic map has a fixed point, then under the condition that $M^{m}\left(t_{0}\right)$ has simple zeros, the $m$-th stroboscopic map of the perturbed system also has a fixed point.

\section{Melnikov subharmonic functions of the parametrically excited pendulum}

For the parametrically excited pendulum, we substitute the vector fields (1) and (A2) into the Melnikov subharmonic function (A4) and obtain

$$
M^{m}\left(t_{0}\right)=\int_{0}^{m T_{p}}-\omega^{\alpha}(t)\left[P \cos \left(\Omega_{p} t\right) \sin \left(\theta^{\alpha}-\phi\right)+\beta \omega^{\alpha}\right] \mathrm{d} t .
$$

Substituting Eq. (2), subject to the resonance condition (A3), into Eq. (A5), we have

$$
\begin{aligned}
M^{m}\left(t_{0}\right)= & -4 k^{2} \beta I_{1}-4 k^{2} P \cos \phi I_{2}+2 k P \sin \phi I_{3} \\
& -4 k^{3} P \sin \phi I_{4},
\end{aligned}
$$

where

$$
\begin{aligned}
I_{1} & =\int_{0}^{m T_{p}} \operatorname{cn}^{2}(t, k) \mathrm{d} t \\
I_{2} & =\int_{0}^{m T_{p}} \cos \left[\Omega_{p}\left(t+t_{0}\right)\right] \operatorname{cn}(t, k) \operatorname{sn}(t, k) \mathrm{dn}(t, k) \mathrm{d} t, \\
I_{3} & =\int_{0}^{m T_{p}} \cos \left[\Omega_{p}\left(t+t_{0}\right)\right] \operatorname{cn}(t, k) \mathrm{d} t, \\
I_{4} & =\int_{0}^{m T_{p}} \cos \left[\Omega_{p}\left(t+t_{0}\right)\right] \operatorname{cn}(t, k) \operatorname{sn}^{2}(t, k) \mathrm{d} t .
\end{aligned}
$$

The dissipation term $-\beta\left(\omega^{\alpha}\right)^{2}$ is the one that contributes to the integrand of $I_{1}$. The integrand of $I_{2}$ comes from the contribution of the vertical excitation term

$$
P \cos \phi \cos \left(\Omega_{p}\left(t+t_{0}\right)\right) \sin \left(\theta^{\alpha}(t)\right),
$$

while the integrand of both $I_{3}$ and $I_{4}$ comes from the contribution of an extra torque term that appears in the asymmetric case, $\phi \neq 0$, given by

$$
\tau=-P \sin \phi \cos \left(\Omega_{p}\left(t+t_{0}\right)\right) \cos \left(\theta^{\alpha}(t)\right) .
$$

Imposing that $M^{m}\left(t_{0}\right)$ in Eq. (A6) has simple zeros allows us to calculate the loci of saddle-node bifurcations generating a subharmonic periodic orbit, i.e., the minimum values $R^{m}$ such that for $P>R^{m}$, we might observe stable oscillatory motion of period $m T_{p}$ in the parameter space.

By integrating Eq. (A7), we have

$$
I_{1}=\frac{4}{k^{2}}\left[E(k)-k^{\prime 2} K(k)\right]
$$

where $k^{\prime 2}=1-k^{2}$ is the complementary elliptic modulus. Expressions $I_{2}, I_{3}$, and $I_{4}$ can be solved by expanding the Jacobi elliptic functions in the Fourier series and using the orthogonality properties between trigonometric functions. To compute $I_{2}$, we first use

$$
\mathrm{d}\left[\mathrm{cn}^{2}(t)\right]=-2 \mathrm{cn}(t) \operatorname{sn}(t) \mathrm{dn}(t) \mathrm{d} t
$$

and integrate by parts. Then, substituting the resonance condition (A3) into the expression for the Fourier expansion of $\mathrm{cn}^{2}(t, k)$ and using orthogonality relations, we end up with

$$
I_{2}= \begin{cases}\frac{\pi \Omega_{p}^{2}}{k^{2}} \operatorname{csch}\left[\Omega_{p} K^{\prime}(k)\right] \sin \left(\Omega_{p} t_{0}\right), & \text { if } m \text { even, } \\ 0, & \text { otherwise }\end{cases}
$$

where $K^{\prime}(k)=K\left(k^{\prime}\right)$. Now, let us compute $I_{3}$. Proceeding the same way as before, after some calculations, we have

$$
I_{3}= \begin{cases}\frac{2 \pi}{k} \operatorname{sech}\left[\Omega_{p} K^{\prime}(k)\right] \cos \left(\Omega_{p} t_{0}\right), & \text { if } m \text { odd } \\ 0, & \text { otherwise. }\end{cases}
$$

Finally, we compute $I_{4}$, which is the most tricky term. We start by writing the product of the Fourier series for $\mathrm{cn}(t, k)$ and $\operatorname{sn}^{2}(t, k)$, substituting the resonance condition (A3). In order to perform the integration, we must compute the integration of the product of three cosines with different arguments. With 


$$
C C=2\left[1-\frac{E(k)}{K(k)}\right] \operatorname{sech}\left(\Omega_{p} K^{\prime}(k)\right),
$$

after cumbersome calculations, we have

$$
I_{4}= \begin{cases}\frac{\pi}{k^{3}}\left(C C-\frac{\pi^{2} S}{K^{2}(k)}\right) \cos \left(\Omega_{p} t_{0}\right), & \text { if } m \text { odd } \\ 0, & \text { otherwise }\end{cases}
$$

where $S$ is given by

$$
\begin{aligned}
S= & \sum_{l=0}^{\infty} \frac{a_{+}(l)}{2} \operatorname{csch}\left[\frac{a_{+}(l)}{m} \Omega_{p} K^{\prime}(k)\right] \times \operatorname{sech}\left[\frac{b(l)}{m} \Omega_{p} K^{\prime}(k)\right] \\
& +\sum_{\substack{l=0 \\
l \neq \frac{m-1}{2}}}^{\infty} \frac{a_{-}(l)}{2} \operatorname{csch}\left[\frac{a_{-}(l)}{m} \Omega_{p} K^{\prime}(k)\right] \\
& \times \operatorname{sech}\left[\frac{b(l)}{m} \Omega_{p} K^{\prime}(k)\right]
\end{aligned}
$$

where $a_{+}(l)=2 l+1+m, a_{-}(l)=2 l+1-m$, and $b(l)=2 l+1$.

\section{Odd and even subharmonic oscillations}

Now, we substitute Eqs. (A9)-(A12) in the expression to the Melnikov integral in Eq. (A6). If $m$ is even, then $I_{3}=I_{4}=0$, and simple zeros of $M^{m}\left(t_{0}\right)$ are due to the term $I_{2}$, which comes from the vertical excitation component. In this case, $R_{\text {even }}^{\mathrm{m}}$ is given by

$$
R_{\text {even }}^{\mathrm{m}}\left(\Omega_{p}\right)=\frac{4 \beta\left[E(k)-k^{\prime 2} K(k)\right]}{\pi \cos \phi \Omega_{p}^{2}} \sinh \left(\Omega_{p} K^{\prime}(k)\right) .
$$

This result agrees with Ref. 8. On the other hand, if $m$ is odd, $I_{2}=0$, and $M^{m}\left(t_{0}\right)$ can only have simple zeros if $\sin \phi \neq 0$, that is, if the additional torque in Eq. (A8) is nonidentically null. Then, $R_{\text {odd }}^{\mathrm{m}}$ is given by

$$
R_{\text {odd }}^{m}\left(\Omega_{p}\right)=\frac{4 \beta\left[E(k)-k^{\prime 2} K(k)\right]}{\pi \sin \phi\left\{\left[\frac{2 E(k)}{K(k)}-1\right] \operatorname{sech}\left(\Omega_{p} K^{\prime}(k)\right)+\frac{\pi^{2} S}{K^{2}(k)}\right\}},
$$

where $S$ is given by Eq. (A13). Note that the elliptic modulus $k$ is determined from $\Omega_{p}$ through the resonance condition, and it is worth noticing that for $\Omega_{p}>m$, there is no $k$ such that Eq. (A3) is satisfied.

${ }^{1}$ J. C. Sartorelli, Phys. Rev. B 45, 10779-10872 (1992).

${ }^{2}$ J. C. Sartorelli, S. Isotani, J. A. Ochi, W. Sano, and A. Piccini, Chem. Phys. Lett. 57, 608-610 (1978).

${ }^{3}$ A. A. Galkin, A. Yu. Kozhukhar', and G. A. Tsintsadze, Zh. Eksp. Teor. Fiz. 70, 248-254 (1976).

${ }^{4}$ T. N. Nogueira, J. C. Sartorelli, M. P. Pato, and C. Ellegaard, Phys. Rev. E 78, 055201(R) (2008).

${ }^{5}$ P. L. Kapitza, Sov. Phys. JETP 21, 588-592 (1951) [Usp. Fiz. Nauk 44, 7-15 (1951)].

${ }^{6}$ J. C. Sartorelli and W. Lacarbonara, Nonlinear Dyn. 69, 1679-1692 (2012).

${ }^{7}$ G. Depetri, J. C. Sartorelli, B. Marin, and M. S. Baptista, Phys. Rev. E 94, 012202 (2016).

${ }^{8}$ B. P. Koch and R. W. Leven, Physica D 16, 1 (1985).

${ }^{9}$ M. A. F. Sanjuán, Chaos Solitons Fractals 9, 995 (1998).

${ }^{10}$ A. Belyakov and A. P. Seyranian, Nonlinear Dyn. 77, 1617 (2014).

${ }^{11}$ K.-H. Kwek and J. Li, Int. J. Nonlinear Mech. 31, 277 (1996).

${ }^{12}$ M. J. Clifford and S. R. Bishop, J. Aust. Math. Soc. B 37, 309 (1996).

${ }^{13}$ P. F. Byrd and M. D. Friedman, Handbook of Elliptic Integrals for Engineers and Physicists, 1st ed. (Springer, Berlin, 1954).

${ }^{14}$ Y. Kuznetsov, Elements of Applied Bifurcation Theory, 2nd ed. (SpringerVerlag, New York, 1998).

${ }^{15}$ E. J. Doedel, "AUTO: A program for the automatic bifurcation analysis of autonomous systems," Congr. Numerantium 30, 265 (1981).

${ }^{16}$ G. A. Leonov, N. V. Kuznetsov, and V. I. Vagaitsev, Physica D 241, 1482-1486 (2012).

${ }^{17}$ E. J. Doedel and B. E. Oldeman, "Auto-07p: Continuation and bifurcation software for ordinary differential equations," Technical Report, Concordia University, Canada (2017).

${ }^{18}$ J. Guckenheimer and P. Holmes, Nonlinear Oscillations, Dynamical Systems, and Bifurcations of Vector Fields, 1st ed. (Springer-Verlag, New York, 1983).

${ }^{19}$ S. Wiggins, Introduction to Applied Nonlinear Dynamical Systems and Chaos, 2nd ed. (Springer-Verlag, New York, 2003). 